\title{
Impulsivity and Time of Day: Is Rate of Change in Arousal a Function of Impulsivity?
}

\author{
Kristen Joan Anderson and William Revelle
}

\begin{abstract}
Impulsivity has been interpreted as a stable mediator of rate of change in arousal states. To test this hypothesis, 129 Ss differing in impulsivity were given placebo or caffeine at 9:00 a.m. or 7:30 p.m. Recognition memory was tested for the last 20 items from 2 lists of 24 items and 2 lists of 80 items. Scores from this paradigm reflect sustained attention and are thus sensitive to changes in arousal. A 4-way interaction among impulsivity, time of day, drug, and prior stimuli $(p<.05)$ indicated that for those given placebo, recognition memory for long and late lists was poorer the higher the impulsivity in the morning; this pattern reversed in the evening. Caffeine reduced recognition errors. These results indicate that impulsivity is not a stable predictor of rate of change in arousal states. Instead, susceptibility to attentional lapses is mediated by impulsivity-related phase differences in diurnal arousal rhythms.
\end{abstract}

Impulsivity is one of the dimensions of individual differences frequently identified by theorists concerned with the biological bases of personality. Although the appropriate theoretical interpretation of impulsivity is a matter of ongoing debate, many models either explicitly or implicitly posit a relationship to arousal (e.g., Barratt \& Patton, 1983; H. J. Eysenck \& Eysenck, 1985; Gale, 1987; Revelle, Anderson, \& Humphreys, 1987; Schalling, Edman, \& Ảsberg, 1983; Strelau, 1987; Zuckerman, 1983). Arguments that impulsivity is linked to arousal can be traced largely to Hans Eysenck (1967), who proposed that (a) there are genetically influenced differences in basal arousal levels, (b) all individuals experience maximally positive hedonic tone at intermediate arousal levels, and (c) individuals who are chronically underaroused develop patterns of behavior designed to increase their arousal. Because social, spontaneous, and risky behaviors (for example) typically afford greater arousal potential than solitary, planned, or safe endeavors, Eysenck proposed that phenotypically extraverted behavior patterns tend to develop among those whose basal arousal levels are low. This theory provided an explanatory link between evidence of the heritability of extraversion and data suggesting differences between introverts and extraverts in a variety of laboratory phenomena.

Kristen Joan Anderson and William Revelle, Department of Psychology, Northwestern University.

This research was supported in part by contracts MDA903-90-C0108 and MDA903-93-K-0008 from the United States Army Research Institute to William Revelle and Kristen Joan Anderson. The views, opinions, and findings contained in this article are ours and should not be construed as an official Department of the Army position, policy, or decision, unless so designated by other official documentation.

We wish to thank Robert Calderon, Debra Janiszewski, Kimberly Moy, Kathryn Rosenthal, Tina Rovick, Vicky Singh, Sara Wilcox, and Peggy Wu for their assistance in collecting and scoring data.

Correspondence concerning this article should be addressed to Kristen Joan Anderson, Department of Psychology, Northwestern University, Evanston, Illinois 60208 .
In brief, Eysenck proposed that the basal arousal level of introverts is higher than that of extraverts and, as a corollary, that the two primary subtraits of extraversion, sociability and impulsivity, are also negatively related to arousal.

The term arousal is used here to refer to a state involving nonspecific physiological activation and the nondirectional component of alertness. Reflected in experiences of alertness, peppiness, and liveliness, it varies within individuals from very low levels characteristic of sleep to very high levels associated with great excitement or panic. More specifically, we use the term arousal to denote a hypothetical construct representing the net result of a variety of processes that mediate activation, alertness, and wakefulness. As an abstraction, it reflects several electrocortical, autonomic, and behavioral mechanisms, but it is not synonymous with any one of them. Despite the difficulties that follow from the concept of a generalized nondirectional energizer, as a hypothetical construct, arousal has pragmatic usefulness in organizing a wide array of empirical phenomena (cf. Anderson, 1990; Claridge, 1987; Gale \& Eysenck, 1992; Hebb, 1955; Humphreys \& Revelle, 1984).

Substantial evidence has been presented in support of the argument linking introversion to arousal (see, e.g., reviews by H. J. Eysenck \& Eysenck, 1985, and Stelmack, 1981), but recent research suggests that revisions to Eysenck's model are necessary. First, there is mounting evidence that it is impulsivity, rather than sociability or extraversion, that is arousal related (e.g., Anderson, in press; Bowyer, Humphreys, \& Revelle, 1983; Campbell, 1992; H. J. Eysenck \& Levey, 1972; Frcka \& Martin, 1987; Loo, 1980; O'Gorman \& Lloyd, 1987; Revelle, Humphreys, Simon, \& Gilliland, 1980; Schalling et al., 1983; Stenberg, 1992).

Moreover, research suggests that the personality-arousal relationship is mediated by phase differences in diurnal arousal rhythms (e.g., Blake, 1967; Revelle et al., 1980). In general, arousal increases during the morning, levels off or decreases slightly through the afternoon, and then declines during the evening; extraverts lag behind introverts in this pattern. Again, al- 
though some inconsistent findings have been reported (e.g., Larsen, 1985; Wilson, 1990), data suggest a greater role for impulsivity than sociability in mediating this relationship between personality and diurnal arousal rhythms (e.g., M. W. Eysenck \& Folkard, 1980; Matthews, 1987b; Neubauer, 1992; Revelle et al., 1980; Zuber \& Ekehammar, 1988). Thus, the available data suggest that high-impulsive subjects are less aroused than lowimpulsive subjects during the morning, but that the reverse is true in the evening, when high-impulsive subjects are more aroused than low-impulsive subjects.

Taken together, these data present serious difficulties for H. J. Eysenck's theoretical model, an issue discussed more fully elsewhere (e.g., Revelle et al., 1987; Revelle \& Anderson, 1992; H. J. Eysenck \& Eysenck, 1985; Gray, 1981). Central to the present study are questions regarding the basis for the apparently complex relationship between impulsivity and arousal. These prior findings force rejection of any simple notion of a direct relationship between impulsivity (or extraversion) and basal arousal levels. Instead, it is possible that impulsivity is a stable predictor of rate of change in arousal states (Revelle, 1983; Revelle et al., 1987). Such a relationship could be found if arousability is a negative function of impulsivity (so that low impulsives become more aroused than high impulsives to equivalent stimuli) $)^{1}$ or if rate of decay of arousal is a positive function of impulsivity (so that high impulsives lose arousal more rapidly than low impulsives). We previously argued that it is this rate of change in arousal states that leads to both impulsivity and to diurnal rhythm shifts:

Nonimpulsives would build up arousal faster than impulsives, becoming alert sooner in the morning. After several hours, high and low impulsives would achieve the same arousal level, but impulsives would seek new stimulation constantly to maintain the arousal. By evening, nonimpulsives, who have been highly aroused for much of the day, would be fatigued and cease to seek arousal. Arousal would decay, and the nonimpulsive would retire for the evening. Impulsives would not have been as highly aroused for as long and would not be fatigued yet. Thus impulsives would still want to maintain a high arousal level and continue to seek stimulation. Eventually fatigue would set in and even impulsives would call it a night. (Revelle et al., 1987, p. 22)

Replacing the Eysenckian concept of stable differences in basal arousal with the proposition that impulsivity reflects stable differences in rate of change in arousal states would thus allow accommodation of the available evidence indicating impulsivity-related differences in diurnal arousal rhythms. At least two lines of research are relevant to this hypothesis: studies linking personality to sustained attention and those addressing individual differences in orienting responses. As reviewed below, this evidence offers some indirect, albeit incomplete, support for this interpretation of impulsivity.

Arguments linking personality to sustained attention follow from a broad base of empirical evidence involving a variety of tasks in which attentional processes are central, that is, tasks such as simple reaction time tasks, simple arithmetic, or letter cancellation, in which subjects are required to process a stimuIus, associate an arbitrary response to that stimulus, and execute that response. Studies of performance on such tasks have yielded converging results from manipulations including drugs, exercise, heat, and noise, as well as state and trait assessments with presumed relevance to arousal. These parallels led several investigators to propose, as part of their broader models of motivation and performance, that arousal facilitates attentional processing, and sustained attention in particular (e.g., Broadbent, 1971; H. J. Eysenck \& Eysenck, 1985; Humphreys \& Revelle, 1984).

If impulsivity is linked to rate of change in arousal states, then in comparison with low impulsives, high impulsives should have greater difficulty sustaining attention. As noted above, there are two versions of this hypothesis linking impulsivity to arousal states, one positing a direct relationship to rate of decay of arousal and the other positing a negative relationship to rate of increase in arousal. If arousal is crucial to sustaining attention and if high impulsives lose arousal more quickly, as suggested by the rate of decay of arousal hypothesis, then high impulsives will experience more attentional lapses over time than low impulsives. Similar predictions follow from the hypothesis linking impulsivity to rate of increase in arousal states: If in comparison with high impulsives, low impulsives are more aroused by stimuli, or if their arousal increases more rapidly, then their greater arousal would facilitate sustained attentional processing and, again, attentional lapses would be more frequent among high impulsives than among low impulsives. Thus, although there are important distinctions between these models in terms of the mechanism identified as responsible for individual differences in rate of change in arousal, the implications of either position for sustained attention are equivalent.

A number of investigators have examined the influence of extraversion on sustained attention in vigilance or continuous performance tasks. A recent meta-analysis of the vigilance literature by Koelega (1992), which highlights methodological difficulties with much of the available research, concluded that the association between extraversion and vigilance decrements, although reliable, is very weak: The estimated effect size of the relationship between extraversion and vigilance decrements as indexed by change in hit rates was $r=.05$. No other performance measure was reliably associated with extraversion over time.

We argue that a central reason for the apparent weakness of the relationship between personality and vigilance decrements is that the crucial variable is impulsivity, not extraversion. If impulsivity is indeed the critical individual-difference dimension, then studies comparing introverts and extraverts would sometimes (but not always) indicate reliable differences (because impulsivity and sociability are moderately correlated); moreover, the differences that are observed would tend to be weaker than those that would be evident if subjects were differentiated on the basis of impulsivity. Koelega's observation that an association between extraversion and vigilance decrements was strongest when extreme groups were used $(r=.27)$ could simply reflect the greater power of that type of design, but it is also true that selecting groups who are extreme in terms of extraversion simultaneously selects for impulsivity, even if the

\footnotetext{
${ }^{1}$ Several extraversion researchers have similarly proposed that introverts and extraverts may differ not in basal arousal levels, but rather in arousability (e.g., Smith, 1983; Stelmack \& Geen, 1992).
} 
measure of extraversion does not include impulsivity content (again, because sociability and impulsivity are positively correlated).

Koelega's review also addressed evidence regarding time of day effects on this relationship. The most glaring problem is that very few researchers have considered the possible role of diurnal arousal variations, neither controlling for nor even reporting the time at which testing occurred. A notable exception is a study by Colquhoun (1960) that included assessment at several times of day and suggested that introverts were better at vigilance than extraverts in the morning, but extraverts were better than introverts in the afternoon. Koelega stressed several criticisms of Colquhoun's project and concluded that time of day does not mediate the extraversion-vigilance relationship. Given both the paucity of available evidence and the potentially critical distinction between impulsivity and extraversion, the question of the impact of time of day on the personality-vigilance relationship must be considered unanswered.

Similar methodological limitations pertain to research on the orienting response. H. J. Eysenck's model (1967) predicted that introverts would show more pronounced orienting responses to stimuli and would also take longer to habituate to them. Research on orienting responses thus bears on hypotheses linking personality to arousability, rate of decay of arousal, or both. As reviewed by H. J. Eysenck and Eysenck (1985), O'Gorman (1983), and Stelmack (1981), a considerable body of evidence is consistent with the hypothesis that extraverts habituate more rapidly than introverts, although inconsistent findings have been reported. Data regarding the magnitude of orienting responses suggests that with moderate-intensity stimuli, introverts show larger skin conductance responses than extraverts; this pattern does not typically emerge with low- or high-intensity stimuli (Stelmack, 1981; Stelmack \& Geen, 1992). As a rule, these studies of orienting responses have examined extraversion rather than impulsivity, and time of day has rarely been reported or controlled. Thus, again, the question of the relationship of impulsivity to rate of change in arousal states remains unanswered.

Taken together, results of research on vigilance decrements and orienting responses provide some limited, but obviously indirect, support for a relationship between impulsivity and rate of change in arousal states. Insofar as this evidence derives primarily from studies conducted during morning or afternoon hours, it is not clear whether these findings will generalize to the evening. A strong test of the hypothesis regarding impulsivity and rate of change in arousal states requires a comparison of high and low impulsives at both morning and evening times on a task sensitive to changes in arousal over time.

One such task requires sustained attention to stimulus materials tested later in a recognition memory paradigm. Previous research by Bowyer et al. (1983) demonstrated the potential usefulness of this task, which they adapted from Underwood (1978). Intrigued by Schulman's (1974) results, which indicated that the positive association between list length and errors in recognition memory was not due to retroactive interference, Underwood (1978) explored the possible role of proactive interference. Subjects completed forced-choice recognition memory tasks after lists of $24,40,60$, and 80 items. In each case, subjects were tested on only the last 20 items, thus equating retention intervals; subjects were not informed of this restriction. Theoretical analyses of proactive interference suggested that with increasing list length, the resultant increase in potential associative links (both semantic and orthographical/acoustical) would produce a corresponding increase in recognition errors. Underwood's methods allowed careful analyses of error patterns, and although error rates did increase reliably with list length, his results failed to support the hypothesis that the higher error rates for longer lists were due to interitem associations. Instead, Underwood advocated an attentional explanation, proposing that attentional lapses were more likely as the number of previous stimuli increased.

If attentional lapses are responsible for the association between list length and error rates in supraspan recognition memory paradigms, and if arousal facilitates sustained attention, then increases in arousal should enhance recognition memory. Bowyer et al. (1983) pursued that possibility in a replication and extension of Underwood's study. Arousal was manipulated through administration of caffeine. In addition, impulsivity was assessed; because all of their subjects were tested in the morning (9:00 a.m.), it was assumed that high impulsives were less aroused than low impulsives. Subjects completed two-alternative forced-choice recognition memory tasks after each of four lists of words; these lists included, in order, 24, 80, 80, and 24 words. Following Underwood (1978), Bowyer et al. tested subjects for only the last 20 words of each list.

Results indicated that error rates increased across the four lists. More crucial was the predicted finding that this decrement in performance over time was reliably greater for high-impulsive subjects than for low-impulsive subjects. Caffeine was associated with fewer errors overall and also with a reduced susceptibility to increasing errors across trials, but the interaction between drug and trial was only marginally significant. Bowyer et al. argued that the parallels between the effects of impulsivity and caffeine on recognition memory within their study and the parallels between their results and findings of other research on attentional performance suggested that a common mechanism was involved, namely, that arousal facilitates sustained attention. As they noted, however, the pattern of results was not fully consistent with their predictions. In particular, parallel effects of arousal variables on list length had been expected, but not confirmed.

The present study is a replication and extension of the experiment reported by Bowyer et al. (1983): High- and low-impulsive subjects were tested in the morning or in the evening after consuming caffeine or placebo. Recognition memory was examined as a function of number of previous stimuli, defined by both list length ( 24 or 80 items) and number of prior lists. This paradigm permitted a test of two hypotheses regarding impulsivity and arousal. As reviewed above, prior research led to a rejection of the hypothesis of a stable relationship between impulsivity and arousal states. The trait of impulsivity could, however, be related to either stable differences in rate of change in arousal states or to stable differences in diurnal arousal rhythms.

1. The first possibility is that regardless of phase differences in diurnal arousal rhythms, impulsivity is associated with stable 
differences in rate of change in arousal states. In this case, highimpulsive subjects should be less able to sustain arousal-and hence less able to sustain attention-over time than low-impulsive subjects, and this difference should be found at all times of day. As already noted, this prediction follows whether the difference in rate of change in arousal states involves rate of increase or rate of decay of arousal. Thus, each model regarding rate of change in arousal states predicts that in both morning and evening sessions, high-impulsive subjects should make more recognition errors than low-impulsive subjects as the number of prior stimuli increases.

2. An alternative hypothesis is that impulsivity is related to phase differences in diurnal arousal rhythms, and it is these diurnal variations in arousal (and not a stable attribute of impulsivity) that determine the capacity to sustain attention. This model predicts that performance on tasks that are facilitated by arousal will reveal a crossover interaction among time of day, impulsivity, and demands for sustained attention: Recognition memory for long and later lists would thus be expected to be poorer for high impulsives than for low impulsives in the morning, but poorer for low impulsives than for high impulsives in the evening.

Both of these hypotheses posit an association between impulsivity and arousal and between arousal and performance on this task. To strengthen interpretation in terms of arousal through convergent validation (cf. Cook \& Campbell, 1979; Cronbach \& Meehl, 1955), arousal was also manipulated through administration of caffeine. Extensive research with this central nervous system stimulant (reviewed by Gilbert, 1976, James, 1991, Lieberman, 1992, and Rall, 1980) is consistent with an arousal interpretation of the effects of the dose we used. It was predicted that caffeine would facilitate sustained attention, and would thus be associated with better recognition memory than placebo as the number of prior stimuli increases.

To summarize our design, subjects differing in impulsivity were given either a placebo or caffeine at either 9:00 a.m. or 7:30 p.m. Following Bowyer et al. (1983), they were then shown four lists of words with (in order) 24, 80, 80, and 24 words. After each list, recognition memory was tested for the last 20 words only, thus equating retention intervals. Several predictions were tested:

1. It was assumed that recognition scores from this paradigm would reflect the subject's ability to sustain attention during the stimulus presentation phase of each trial and across trials. Recognition scores were thus expected to be lower for 80 -item and later lists in comparison with the first, short list.

2. It was assumed that increased arousal would facilitate sustained attention. Caffeine, which produces an increase in arousal, was therefore expected to be associated with fewer recognition memory errors than placebo. Beneficial effects of caffeine were expected to be most pronounced when the number of previous stimuli (and thus the demand for sustained attention) was high, that is, for the long and late lists.

3. The primary purpose of this study was to examine two different hypotheses regarding impulsivity: If impulsivity is related to stable differences in rate of change in arousal states, then high impulsives given placebo would show poorer recognition for 80-item and later lists than low impulsives given pla- cebo, and this would be found in both morning and evening sessions. In contrast, if impulsivity is related to phase differences in diurnal arousal rhythms, but not to stable differences in rate of change in arousal states, then impulsivity and time of day would interact: In the morning, high-impulsive subjects given placebo would recognize fewer items from long and later lists than their low-impulsive counterparts. In the evening, the reverse would be true. Thus, these two hypotheses about impulsivity yield similar predictions for subjects given placebo and tested in the morning, but opposite predictions for subjects given placebo and tested in the evening.

\section{Method}

\section{Subjects}

Subjects included 157 students taking introductory psychology at Northwestern University; they received partial course credit for participating. Individual differences were assessed with the Eysenck Personality Inventory (EPI; H. J. Eysenck \& Eysenck, 1964), using the impulsivity subscale identified by Revelle et al. (1980). Scores on this scale, which emphasizes spontaneity and rapidity of cognitive and behavioral activity, have repeatedly been shown to mediate performance as expected on the basis of predictions from arousal theory (e.g., Anderson, in press; Anderson \& Revelle, 1982; Bowyer et al., 1983; Revelle et al., 1980). The distribution of impulsivity scores $(M=4.61, S D=2.07)$ was commensurate with other samples. Alternative EPI impulsivity scores were available for many of the subjects included in analyses. Comparison with scores obtained under conditions in which the subjects spent an hour completing various personality questionnaires yielded a test-retest reliability of $.72(n=124)$; comparison with scores obtained in other experiments in our lab indicated a test-retest reliability of $82(n=95)$.

Equipment malfunctions prevented use of data from 13 of these subjects. In addition, 1 subject became ill during the experimental session and 5 had consumed caffeine shortly before participating. One subject grossly violated task directions and 8 subjects displayed excessively low recognition rates (less than $56 \%, 3 S D$ below mean levels), suggesting failure to comply with experimental instructions. Data analyses are thus based on 129 subjects. $^{2}$

Assignment to time of day and drug conditions was random and was determined before the experimental session began. Of the subjects included in analyses and tested at 9:00 a.m., 29 received placebo and 34 caffeine; of those tested at 7:30 p.m., 32 received placebo and 34 caffeine. Impulsivity scores did not differ reliably across experimental conditions.

\section{Materials}

Caffeine versus placebo. Subjects received either placebo (flat quinine water) or caffeine citrate ( $4 \mathrm{mg}$ caffeine per $\mathrm{kg}$ body weight) dissolved in an orange-flavored breakfast drink.

Memory task. The stimulus set, which supplemented the list used by Underwood (1978) by four words, included 288 four-letter words that varied greatly in frequency. Four lists of words, two with 24 items and two with 80 items, were randomly selected and randomly ordered, with the remaining items used as distractors in two-alternative, forcedchoice recognition tests. Stimuli were presented in the same order to all

\footnotetext{
${ }^{2}$ With the exception of the one subject who clearly violated task instructions by circling both items in some pairs and neither item in others, the pattern of means was not affected by exclusion of these subjects.
} 
subjects. The recognition tests probed only the last 20 items of each list; word pairings and pair order for the recognition tests were randomly determined.

\section{Procedure}

Subjects were scheduled by telephone for a session at the time of day to which they had been randomly assigned; they were not informed that sessions were also being run at an alternative time. All subjects were asked to avoid caffeine or any other psychoactive drug for at least $6 \mathrm{hr}$ beforehand.

On their arrival, subjects were provided with a consent form that indicated possible side effects of caffeine and screened for contraindicated medical conditions. All subjects consented and were given the beverage containing either caffeine or placebo using double-blind procedures.

Subjects then completed several questionnaires, including the EPI and a set of questions regarding typical and immediately prior caffeine consumption. When $23 \mathrm{~min}$ had elapsed since drug administration, subjects completed several state measures, including the 40 items from Thayer's Activation-Deactivation Adjective Checklist (AD-ACL; 1986) rated on a 0 to 3 scale.

Instructions for the recognition task were then given: Subjects were informed that they would see several lists of words that varied in length, that after each list they would be shown a set of pairs of words and asked to indicate which word from each pair was on the list; and that not all items on the list would be tested. They were told not to leave any pairs unmarked, guessing if necessary. Subjects were not told how many items were in any list, how many items would be probed, or that probed items were always from the end of the list. Furthermore, to minimize the possibility that anticipation of the end of the task would influence performance on the last list (cf. Anderson \& Revelle, 1983), two extra trays of slides were placed with the four that were actually used to create the impression that further lists would be presented.

Once any questions were answered, we presented the first list of 24 words using a slide projector. The slides, each having a single word in lowercase letters in the center, were presented at a 2.7 -s rate. Subjects were then given 90 s to complete a printed two-alternative forced-choice recognition task. The two 80 -word lists and the final 24 -word list were presented and tested in like manner. This ordering of lists directly replicated that used by Bowyer et al. (1983)

The AD-ACL and other state measures were then readministered. Finally, subjects were thanked and debriefed. They were urged to refrain from discussing the experiment with other potential subjects.

\section{Results}

\section{Manipulation Check}

AD-ACL scores were subjected to a general linear model analysis with trial (before or after the memory task) as a withinsubjects factor, time of day (9:00 a.m. or 7:30 p.m.) and drug (caffeine or placebo) as categorical between-subjects factors, and impulsivity as a continuous between-subjects factor.

Although the predicted main effect of caffeine was evident, $F(1,121)=11.99, M S_{\mathrm{e}}=160.89, p<.001$, this analysis also indicated an unexpected three-way interaction among trial, time of day, and drug, $F(1,121)=9.47, M S_{\mathrm{e}}=36.44, p<.01$. (See Figure 1.) Inspection of the means indicates that in the evening, caffeine was, as expected, associated with higher levels of self-reported activation than placebo both before and after completing the recognition task. In contrast, caffeine was not associated with higher levels of self-reported activation than pla- cebo immediately before the recognition task among subjects tested in the morning. Their self-reported activation scores were higher with caffeine than with placebo after the task.

\section{Recognition Memory Scores}

Number of correct responses on the recognition tests was subjected to a general linear model analysis with time of day (9:00 a.m. or 7:30 p.m.) and drug (caffeine or placebo) as categorical between-subjects factors and impulsivity as a continuous between-subjects factor. Within-subjects effects were analyzed using planned orthogonal contrasts selected on the basis of our hypotheses.

1. We expected that susceptibility to attentional lapses would increase as both number of prior lists and number of prior items increased. Thus, recognition memory should be poorer for the longer and later lists than for the initial list of 24 items. Our first contrast compared performance on the first list with that on the three later lists (which included both 80-item lists); we refer to this contrast as the effect of prior stimuli.

2. This same logic led to the prediction that performance on the second list of 80 items should be worse than on the first list of 80 items. Our second contrast thus compared these two lists and is referred to as the set effect for long lists.

3. The remaining orthogonal contrast tested the effect of list length by comparing the two 80 -item lists with the final 24-item list. We did not have a specific prediction for this contrast, because performance on the final list reflects both increasing time on task (rendering it more susceptible to attentional lapses than earlier lists) and shorter list length (rendering it less susceptible to attentional lapses than the 80 -item lists). ${ }^{3}$

List effects. Results indicated a significant effect of prior stimuli, $F(1,121)=59.40, M S_{\mathrm{e}}=25.60, p<.001$. Means for the four lists in the order of their presentation (i.e., the first list of 24 , the first list of 80 , the second list of 80 , and the second list of 24) were 19.22, 17.81, 18.21, and 18.37. Thus, as expected, average recognition memory scores were lower for the long and late lists than for the first, short list. The set effect for long lists was also reliable, $F(1,121)=4.63, M S_{\mathrm{e}}=1.85, p<.05$. Contrary to expectation, average scores for the second list of 80

\footnotetext{
${ }^{3}$ Two alternative analyses would have been feasible, one subjecting the list effects to trend analysis, and the other to a factorial design crossing list length with set (first or second). Neither option directly addresses our central hypotheses. Moreover, both ignore the ambiguity of predictions regarding the final list relative to the 80 -item lists. As a result, any of several outcomes from either of these alternative analyses could have been viewed as consistent with our hypotheses. That is, interactions of arousal variables with either linear or quadratic trends could have been interpreted as consistent with our predictions; alternatively, interactions of arousal variables with list length, set, or their interaction could have been interpreted as supporting our model. Recognizing that a priori specification of particular patterns of results is not always possible and that, as a result, a probabilistic bias in favor of one's hypotheses can accrue, we have previously argued (Anderson, in press; Revelle \& Anderson, 1992) for the use of techniques that reduce the number of outcomes that can be construed as consistent with predictions. We thus rejected both the trend and the factorial analytic strategies in favor of comparisons that provided unambiguous tests of our predictions.
} 


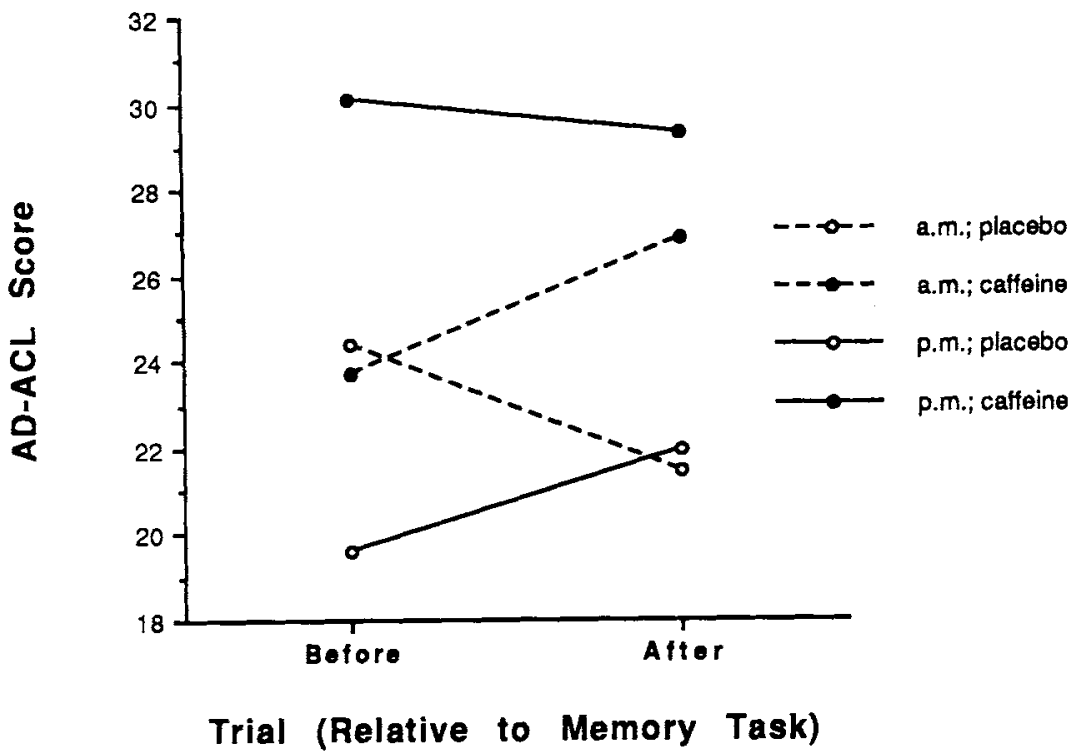

Figure 1. Activation-Deactivation Adjective Checklist (AD-ACL) scores as a function of time of day (9:00 a.m. or 7:30 p.m.), drug (placebo or caffeine), and trial (before or after the recognition memory task).

items were higher than for the first list of 80 items. List length also significantly influenced recognition scores, $F(1,121)=$ $6.00, M S_{\mathrm{e}}=8.60, p<.05$. Average scores were higher for the final list of 24 items than for the two 80 -item lists.

Caffeine effects. It was predicted that caffeine would enhance sustained attention, thus resulting in better recognition memory for 80-item lists and lists late in the experimental session. The main effect of caffeine, $F(1,121)=11.52, M S_{\mathrm{e}}=6.66$, $p<.001$, was significant. More important, caffeine interacted with the prior stimulus effect, $F(1,121)=9.68, M S_{e}=25.60, p$ $<.01$. As shown in Figure 2, the pattern of means indicates that subjects given caffeine recognized more items from the last three lists than did those given placebo.

Impulsivity effects. The crucial question addressed by this study concerned the effects of impulsivity on recognition memory. If the personality dimension of impulsivity is related to stable differences in rate of change in arousal states, then regardless of time of day, high-impulsive subjects given placebo would be more likely than low-impulsive subjects given placebo to show vigilance-like decrements in recognition memory. Thus, impulsivity would interact with prior stimuli, but time of day would not mediate that interaction. In contrast, if the relationship between impulsivity and rate of change in arousal states is not temporally stable, instead depending on diurnal arousal rhythms (which are in turn linked to impulsivity), then susceptibility to vigilance-like decrements would be an interaction of impulsivity with time of day: In the morning, high impulsives given placebo would be more susceptible to vigilance-like decrements; in the evening, it would be the low impulsives given placebo who would be more likely to show this susceptibility.

The crucial finding was of a four-way interaction between impulsivity, time of day, drug, and the prior stimulus effect, $F(1$, $121)=4.07, M S_{\mathbf{e}}=25.60, p<.05$. (See Figure 2.) Inspection of the means confirms the already noted effect of caffeine: At both times of day, subjects given placebo performed more poorly on the three later lists than did those given caffeine. Most important, for those given placebo, a crossover interaction of impulsivity with time of day was evident: Among those given placebo in the morning, the more impulsive the subject, the lower the recognition scores for the long and late lists $(r=-.25)$. In the evening, the impulsivity effects for placebo subjects reversed: At this time of day, it was the more impulsive subjects who had the least difficulty with the three later lists $(r=.12)$. For the subjects given caffeine, the correlations between impulsivity and average scores for the long and late lists were .17 in the morning and -.24 in the evening.

Simple effects tests confirmed the reliability of the 3-way interaction among impulsivity, time of day, and caffeine for the later three lists, simple $F(1,121)=4.64, M S_{\mathrm{e}}=7.27, p<.05$. To further clarify this interaction, scores for each list were analyzed separately: On the first list of 24 items, recognition memory scores did not vary reliably as a function of caffeine, time of day, impulsivity, or any of their interactions. On the first list of 80 items, the effect of caffeine approached significance, $F(1,121)$ $=3.18, M S_{\mathrm{e}}=3.81, p<.08$. On each of the last two lists, the triple interaction of drug, impulsivity, and time of day was reliable, $F(1,121)=3.93, M S_{\mathrm{e}}=3.45, p<.05$, for the second $80-$ item list and $F(1,121)=3.95, M S_{\mathrm{e}}=3.29, p<.05$, for the second 24-item list. Similarly, the effect of caffeine was reliable for each of these later two lists, $F(1,121)=11.40, M S_{\mathrm{e}}=3.45$, $p<.001$, for the second 80 -item list, and $F(1,121)=16.31$, $M S_{\mathrm{e}}=3.29, p<.001$, for the second 24-item list.

Finally, results also indicated a lower-order interaction between time of day and prior stimuli, $F(1,121)=4.05, M S_{\mathrm{e}}=$ $25.60, p<.05$. Although there was little difference on the first list of 24 items between subjects tested in the morning ( $M=$ 


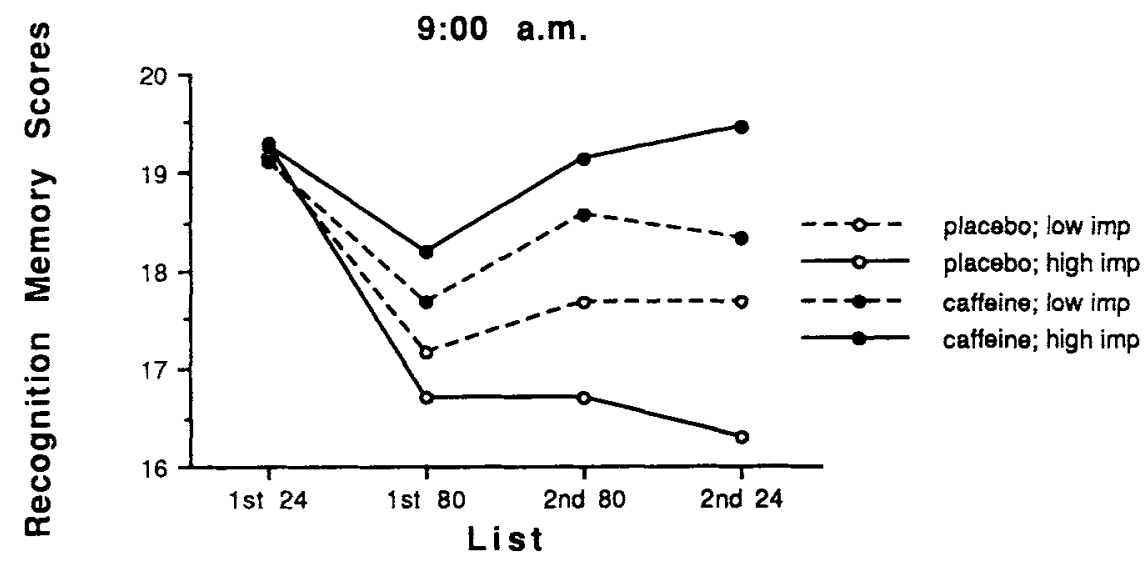

7:30 p.m.

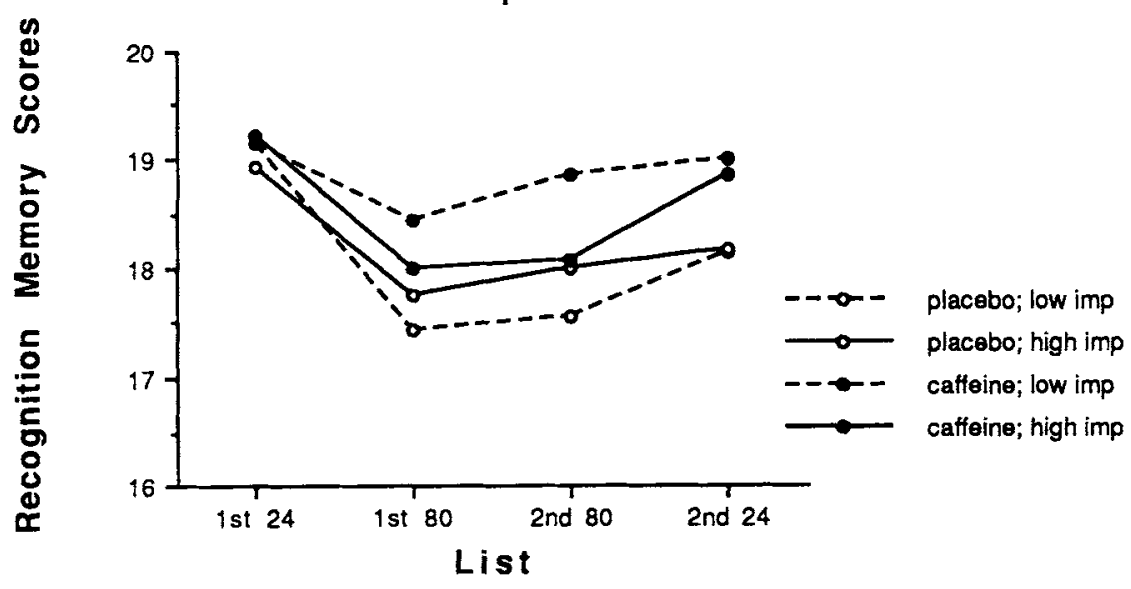

Figure 2. Recognition memory scores as a function of time of day (9:00 a.m. or 7:30 p.m.), impulsivity (imp), drug (placebo or caffeine), and list. Subjects with impulsivity scores greater than 5 were considered high impulsive; those with scores less than 4 , low impulsive.

19.30) and the evening $(M=19.15)$, subjects tested in the evening recognized more items from the long and late lists $(M=$ $18.29)$ than did those tested in the morning $(M=17.95)$.

No other effects, main or interactive, reached standard levels of statistical significance.

Other personality effects. The crucial hypotheses tested in this experiment involved impulsivity. As noted above, we believe that one reason for apparent inconsistencies in the available literature linking personality to performance is that many researchers focus on extraversion or sociability rather than impulsivity. To examine this argument, we also analyzed our data as a function of these personality dimensions.

When sociability (as measured by the EPI subscale identified by Revelle et al., $1980 ; M=7.60, S D=2.63$ ) replaced impulsivity as a continuous variable in the analysis, one effect involving this personality dimension reached standard levels of statis- tical significance: Sociability interacted with caffeine and the list-length effect (i.e., the comparison of the two 80-item lists to the final list of 24 items), $F(1,121)=4.82, M S_{\mathrm{e}}=8.25, p<.05$. (See Figure 3.) For the subjects given placebo, the correlation between sociability and recognition scores was slightly positive: .10 for the 80 -item lists and .06 for the second list of 24 items. For the subjects given caffeine, the correlation was slightly negative for the 80 -item lists $(r=-.10)$, but positive for the second 24-item list $(r=.20)$.

With extraversion $(M=13.57, S D=4.25)$ replacing impulsivity as the personality variable included in the design, the fourway interaction among extraversion, time of day, caffeine, and prior stimuli was statistically significant, $F(1,121)=4.38, M S_{\mathrm{e}}$ $=25.46, p<.05$. The interaction of extraversion, caffeine, and the list length effect was also significant, $F(1,121)=4.25, M S_{e}$ $=8.39, p<.05$. No other reliable effects involved extraversion. 


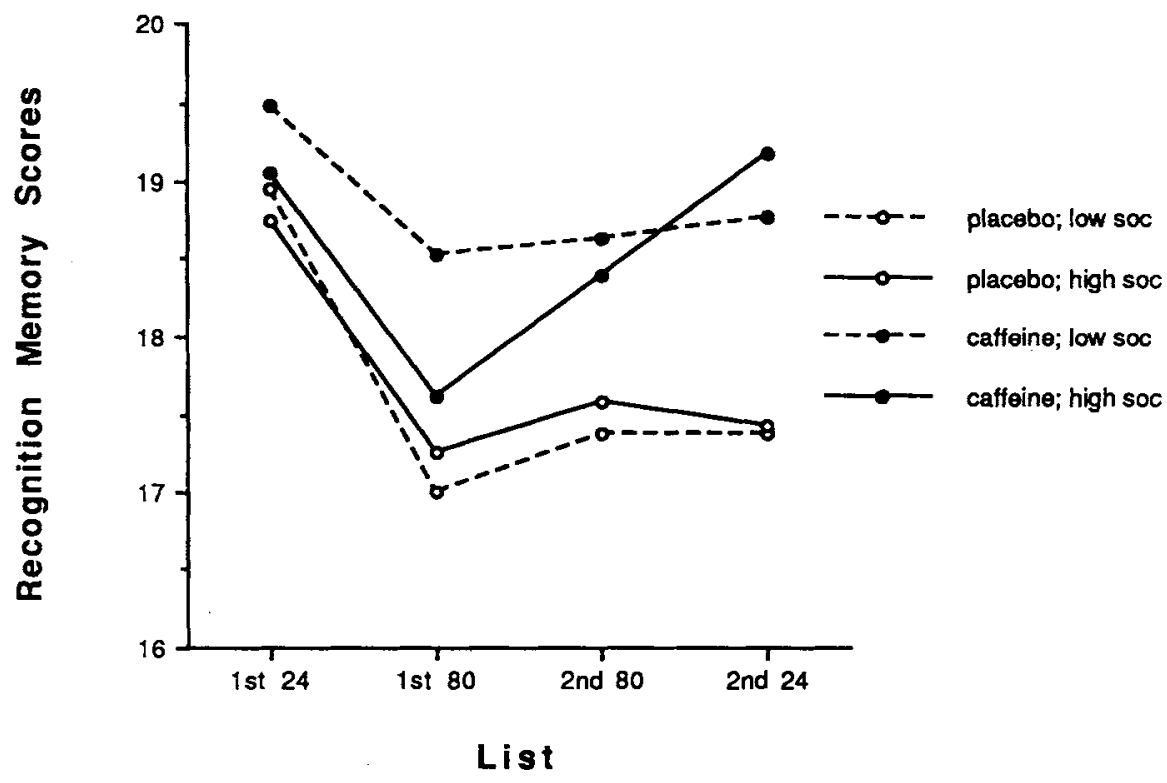

Figure 3. Recognition memory scores as a function of sociability (soc), drug (placebo or caffeine), and list. Subjects with sociability scores greater than 9 were considered high sociable; those with scores less than 7 , low sociable.

\section{Discussion}

The central purpose of this study was to clarify the relationship between impulsivity and arousal. Although a stable relationship between the trait of impulsivity and states of arousal had been rendered implausible by previous research, questions remained regarding the relationship of this personality dimension to arousal states. The trait of impulsivity could be related to stable differences in rate of change in arousal states or, alternatively, to phase differences in diumal arousal rhythms.

Our key finding was a four-way interaction among impulsivity, time of day, drug, and prior stimuli. The pattern of means indicates that (a) regardless of time of day, subjects given placebo recognized fewer words from the 80-item lists and from the second 24-item list than subjects given caffeine and (b) in the absence of caffeine, recognition memory for these long or late lists was poorer the higher the impulsivity in the morning, but better the higher the impulsivity in the evening. This crossover interaction between impulsivity and time of day contradicts the hypothesis that impulsivity is related to stable individual differences in either basal arousal levels or in rate of change in arousal states. Instead, this interaction is consistent with the alternative hypothesis that impulsivity is linked to phase differences in diurnal arousal rhythms, which in turn determine ability to sustain attention.

Relative to placebo, caffeine reliably facilitated recognition memory for long and late lists, as anticipated if arousal enhances sustained attention. Results of our manipulation check unexpectedly indicated that the placebo and caffeine subjects in the morning session did not differ reliably in self-reported activation states in the minutes immediately preceding the first memory task. Thus, it is possible that the beneficial effect of caffeine on later trials in the morning merely reflected an increase in the extent to which the drug had taken effect. It is worth noting, however, that the effects of caffeine on recognition memory across the four lists in the evening are similar to the effects observed in the morning, and data from the evening session indicated large, reliable differences in self-reported activation both before and after the memory task. It is thus difficult to attribute the observed effects of caffeine on the later lists solely to a delayed response to the drug during the morning.

It is also important to stress that despite some lack of clarity regarding the interaction of caffeine with the prior stimuli effect, interpretation of the higher order crossover interaction among impulsivity, time of day, caffeine, and prior stimuli is not ambiguated by the self-report data. Interpretation of this higher order interaction relies on the observed crossover between impulsivity and time of day for late and long lists among the subjects given placebo, which demonstrates that the impact of impulsivity on recognition errors reverses from morning to evening. This pattern suggests that while engaging in a relatively monotonous task in the morning, high impulsives are more susceptible than low impulsives to a reduction in arousal level over time, but in the evening, it is the low impulsives who are more susceptible to this effect. That caffeine minimized recognition memory errors on late lists (when self-report measures suggested reliable differences between placebo and caffeine subjects) provides convergent validation of our interpretation of this higher order interaction in terms of arousal.

One intriguing aspect of the observed effect of caffeine was that in both morning and evening sessions, it was most beneficial to those subjects who, in the absence of caffeine, had the most difficulty with the task. This outcome was not expected. 
(We had predicted that caffeine would be associated with fewer recognition errors, but had not predicted disordinal interactions involving caffeine.) We have argued elsewhere (e.g., Humphreys \& Revelle, 1984; Revelle et al., 1987) that arousal interferes with short-term memory processes and have demonstrated that caffeine increases the time required to scan items in short-term memory within a Sternberg paradigm (Anderson, Revelle, \& Lynch, 1989). Although we believe that the task used in this experiment reflects primarily the influence of attentional processes, it is possible that a weak detrimental effect of arousal on short-term memory is counteracting its beneficial effects on attention for the more aroused subjects, that is, the low-impulsive subjects given caffeine in the morning and the high-impulsive subjects given caffeine in the evening. ${ }^{4}$

We had expected that susceptibility to vigilance-like decrements would increase with both number of previous lists and number of previous items within a list. Thus, we had predicted that performance on the second list of 80 items would be worse than that on the first such list, particularly among subjects given placebo. Our findings were contrary to this hypothesis. Spurious differences in list difficulty, changes in the subject's expectations about the task, or practice effects could have counteracted a detrimental impact of time on task. The design of this experiment does not permit evaluation of these possibilities, although comparison with the pattern of results from Bowyer et al. (1983), who found that errors were somewhat more common on the second than the first list of 80 items, tends to support the list-difficulty interpretation.

As reviewed above, our interpretation of results in terms of arousal and sustained attention draws on previous research: First, Underwood (1978) strongly suggested that recognition memory errors for supraspan lists in the paradigm we used reflect attentional lapses during stimulus presentation. Second, the parallels between performance in this paradigm and in other continuous-performance or vigilance tasks, and convergences across a range of manipulated and observed variables in their impact on performance on such tasks, are most parsimoniously explained by positing a common mechanism, specifically, that arousal facilitates sustained attention (cf. Bowyer et al., 1983; Broadbent, 1971; Humphreys \& Revelle, 1984). These data thus bear on the question of the relationship between personality and vigilance decrements. As discussed above, Koelega's (1992) recent review suggested at best a weak relationship between extraversion and vigilance decrements and questioned the importance of time of day effects. This project joins others (e.g., Matthews, 1987a; Revelle et al., 1980) in suggesting that time of day is a crucial moderator of personality effects on cognitive tasks, with theoretically meaningful arousal-related reversals occurring between morning and evening sessions. Moreover, as we have argued before, impulsivity appears more closely associated with arousal variations and hence performance than sociability or extraversion.

Our analyses in terms of alternative personality measures highlight these conclusions: When we reanalyzed the data with sociability replacing impulsivity as the personality variable, sociability was found to interact with drug and list length. This result indicated that among subjects given placebo, higher sociability was associated with slightly better recognition mem- ory. Among those given caffeine, high sociability was associated with better recognition memory for the second 24 -item list but poorer recognition memory for the two 80 -item lists. According to Eysenck's model, sociability and arousal are negatively associated. The finding that low sociability was associated with better performance only among subjects given caffeine and only on 80-item lists is inconsistent with an arousal interpretation.

Our measure of extraversion (from the EPI) reflects both impulsivity $(r=.81)$ and sociability $(r=.84)$. Thus, it is not surprising that our findings involving extraversion captured the results of analyses based on each dimension separately. Analyses in terms of impulsivity yielded predicted and interpretable results; analyses in terms of sociability yielded unexpected and uninterpretable results; analyses in terms of extraversion yielded a mixture of interpretable and uninterpretable findings. This pattern is consistent with our argument that impulsivity is the crucial personality variable in arousal research, and further, that reliance on measures of extraversion will yield weak or inconsistent evidence regarding underlying relationships. That many measures of extraversion tap sociability but not impulsivity (see, for example, Rocklin \& Revelle, 1981) raises obvious difficulties for research in this domain. ${ }^{5}$

In confirming previous suggestions that (a) impulsivity, not sociability, is the personality dimension that mediates arousalrelated performance effects and (b) impulsivity is related to phase differences in diurnal arousal rhythms, the results of this project raise serious difficulties for several theoretical interpretations of the individual-difference dimension of impulsivity. Models linking impulsivity to stable differences in basal arousal, stable differences in general rate of decay of arousal or habituation, or stable differences in general rate of increase in arousal or arousability are all inconsistent with the observed pattern of results. That is, any model positing a temporally consistent relationship between impulsivity and arousal states or between impulsivity and patterns of change in arousal states is questioned by these data.

Two particular models deserve attention here. First, these data obviously contradict our own previous arguments (e.g., Revelle et al., 1987; Revelle \& Anderson, 1992) that impulsivity is linked to stable differences in rate of change in arousal states. Second, these findings disconfirm interpretation of impulsive

\footnotetext{
${ }^{4}$ This logic would suggest that the effects of arousal might vary across the serial position curve. Although the experiment was not designed to test serial position effects, an additional analysis was conducted to explore this possibility. The 20 words tested from each list were grouped into four sublists in order of stimulus presentation. A Serial Position $\times$ Trial interaction, $F(9,1089)=6.08, M S_{\mathrm{e}}=0.36, p<.001$, indicated that serial position effects varied from list to list. Examination of the pattern of means failed to suggest a recency effect for any list. The interaction between serial position, trial, impulsivity, and caffeine was also significant, $F(9,1089)=2.15, M S_{c}=0.36, p<.05$. The pattern of correlations between impulsivity and recognition memory scores varied across lists and serial positions and was thus not amenable to theoretical interpretation.

${ }^{5}$ Changes in the measurement of extraversion from inclusion of both sociability and impulsivity content to emphasis only on sociability reflects a change in Eysenck's theoretical conceptualization of the structure of personality (e.g., H. J. Eysenck \& Eysenck, 1985).
} 
behaviors in terms of states of arousal per se, thus creating substantial difficulties for H. J. Eysenck's (1967) theoretical explanation of impulsive behavior (cf. Revelle et al., 1987; Revelle \& Anderson, 1992; Gray, 1981). Specifically, our data suggest that temporally consistent patterns of impulsive behavior cannot be attributed to efforts to compensate for either low basal arousal levels or stable tendencies toward low arousal levels. That is, if recognition memory in this supraspan paradigm does reflect the influence of arousal, then our data suggest that high-impulsive subjects are (a) more (not less) aroused than low impulsives in the evening and (b) more (not less) able to sustain arousal than low impulsives in the evening. It is thus difficult to attribute impulsive behavior, which presumably occurs more frequently among high impulsives than low impulsives no matter what the time of day, to either low arousal or a greater rate of change in arousal. Instead, the direction of causality may be from impulsivity to arousal states, rather than from arousal states to impulsivity.

We cannot reject the possibility that impulsivity is related to differences in preferred arousal level, an issue that, as Gale (1981) noted, has received virtually no serious investigation. Similarly, Gray's (1981) hypothesis that impulsivity reflects sensitivity to signals of reward was not directly addressed by this project. Although this study did not offer a test of these models of impulsivity, the evidence for a link between impulsivity and diurnal arousal rhythms suggests that a complete understanding of this personality dimension will require some consideration of arousal states.

That impulsivity is arousal related is strongly supported by the pattern of results from this and other studies of cognitive performance, which have yielded predictable and replicable interactions among impulsivity, arousal manipulations, and task characteristics. Moreover, as discussed elsewhere (e.g., Revelle \& Anderson, 1992), the higher order interactions observed in these studies have eliminated a variety of alternative (i.e., nonarousal related) explanations for the performance effects. For example, although impulsivity is associated with a preference for speed over accuracy, such strategic differences cannot explain the full pattern of results. (Note that the reversal of impulsivity differences from morning to evening in the present study likewise argues against a stable strategic explanation for the observed performance effects.) Similarly, hypotheses of differential sensitivity to caffeine or of performance disruption due to monitoring of unusual states of arousal are eliminated by interactions of impulsivity with time of day.

To summarize, our data suggest that impulsivity is linked to arousal and thereby to performance, but in a complex way: Susceptibility to lapses in attention is a function of arousal, with impulsivity bearing a consistent relationship not to basal arousal levels, and not to rate of change in arousal states, but rather to phase differences in diurnal arousal rhythms. Thus, high impulsives are more susceptible to vigilance-like decrements than low impulsives in the morning, but less susceptible in the evening. This interaction of impulsivity with time of day contradicts hypotheses linking impulsivity to stable differences in basal arousal level (e.g., H. J. Eysenck, 1967) or in rate of change in arousal states (e.g., Revelle et al., 1987) and raise serious difficulties for any model proposing that individual differences in arousal states cause impulsive behavior. Instead, these findings strongly support an association between impulsivity and phase differences in diurnal arousal rhythms.

\section{References}

Anderson, K. J. (1990). Arousal and the inverted-u hypothesis: A critique of Neiss's "Reconceptualizing arousal." Psychological Bulletin, $107,96-100$.

Anderson, K. J. (1994). Impulsivity, caffeine, and task difficulty: A within-subjects test of the Yerkes-Dodson law. Personality and Individual Differences, 16, 813-829.

Anderson, K. J., \& Revelle, W. (1982). Impulsivity, caffeine, and proofreading: A test of the Easterbrook hypothesis. Journal of Experimen tal Psychology: Human Perception and Performance, 8, 614-624.

Anderson, K. J., \& Revelle, W. (1983). The interactive effects of caffeine, impulsivity and task demands on a visual search task. Personality and Individual Differences, 4, 127-134.

Anderson, K. J., Revelle, W., \& Lynch, M. J. (1989). Caffeine, impulsivity, and memory scanning: A comparison of two explanations for the Yerkes-Dodson effect. Motivation and Emotion, 13, 1-20.

Barratt, E. S., \& Patton, J. H. (1983). Impulsivity: Cognitive, behavioral, and psychophysiological correlates. In M. Zuckerman (Ed.), Biological bases of sensation seeking, impulsivity, and anxiety (pp. 77122). Hillsdale, NJ: Erlbaum.

Blake, M. J. F. (1967). Relationship between circadian rhythm of body temperature and introversion-extraversion. Nature, 215, 896-897.

Bowyer, P., Humphreys, M. S., \& Revelle, W. (1983). Arousal and recognition memory: The effects of impulsivity, caffeine, and time on task. Personality and Individual Differences, 3, 41-49.

Broadbent, D. E. (1971). Decision and stress. San Diego, CA: Academic Press.

Campbell, J. B. (1992). Extraversion and noise sensitivity: A replication of Dornic and Ekehammar's study. Personality and Individual Differences, 13, 953-955.

Claridge, G. (1987). Psychoticism and arousal. In J. Strelau \& H. J. Eysenck (Eds.), Personality dimensions and arousal (pp. 133-150). New York: Plenum.

Colquhoun, W. P. (1960). Temperament, inspection efficiency, and time of day. Ergonomics, 3, 377-378.

Cook, T. D., \& Campbell, D. T. (1979). Quasi-experimentation: Design and analysis issues for field settings. Chicago: Rand McNally.

Cronbach, L. J., \& Meehl, P. E. (1955). Construct validity in psychological tests. Psychological Bulletin, 52, 281-302.

Eysenck, H. J. (1967). The biological basis of personality. Springfield, IL: Charles C Thomas.

Eysenck, H. J., \& Eysenck, M. W. (1985). Personality and individual differences: A natural science approach. New York: Plenum.

Eysenck, H. J., \& Eysenck, S. B. G. (1964). Eysenck Personality Inventory. San Diego, CA: Educational and Industrial Testing Service.

Eysenck, H. J., \& Levey, A. (1972). Conditioning, introversion-extraversion and the strength of the nervous system. In V. D. Nebylitsyn \& J. A. Gray (Eds.), Biological bases of individual behavior (pp. 206220). San Diego, CA: Academic Press.

Eysenck, M. W., \& Folkard, S. (1980). Personality, time of day and caffeine: Some theoretical and conceptual problems in Revelle et al. Journal of Experimental Psychology: General, 109, 32-41.

Frcka, G., \& Martin, I. (1987). Is there-or is there not-an influence of impulsiveness on classical eyelid conditioning? Personality and Individual Differences, 8, 241-252.

Gale, A. (1981). EEG studies of extraversion-introversion: What's the next step? In R. Lynn (Ed.), Dimensions of personality: Papers in honour of H. J. Eysenck (pp. 181-207). Oxford, England: Pergamon Press. 
Gale, A. (1987). Arousal, control, energetics, and values: An attempt at review and appraisal. In J. Strelau \& H. J. Eysenck (Eds.), Personality dimensions and arousal (pp. 287-316). New York: Plenum.

Gale, A., \& Eysenck, M. W. (1992). Psychophysiology: A programme for individual differences research? In A. Gale \& M. W. Eysenck (Eds.), Handbook of individual differences: Biological perspectives (pp. 1-18). New York: Wiley.

Gilbert, R. M. (1976). Caffeine as a drug of abuse. In R. G. Gibbons, Y. Israel, H. Kalant, R. E. Popham, W. Schmit, \& R. G. Smart (Eds.), Research advances in alcohol and drug problems (Vol. 3, pp. 47-176). New York: Wiley.

Gray, J. A. (198I). A critique of Eysenck's theory of personality. In H. J. Eysenck (Ed.), A model for personality. Berlin: Springer-Verlag.

Hebb, D. O (1955). Drives and the C.N.S. (Conceptual Nervous System). Psychological Review, 62, 243-254.

Humphreys, M. S., \& Revelle, W. (1984). Personality, motivation, and performance: A theory of the relationship between individual differences and information processing. Psychological Review, 91, 153184.

James, J. E. (1991). Caffeine and health. San Diego, CA: Academic Press.

Koelega, H. S. (1992). Extraversion and vigilance performance: 30 years of inconsistencies. Psychological Bulletin. 112, 239-258.

Larsen, R. J. (1985). Individual differences in circadian activity rhythm and personality. Personality and Individual Differences, 6, 305-311.

Lieberman, H. R. (1992). Caffeine. In A. P. Smith \& D. M. Jones (Eds.), Handbook of human performance: Vol. 2. Health and performance (pp. 49-72). San Diego, CA: Academic Press.

Loo, R. (1980). Role of primary personality factors in the perception of traffic signs and driver violations and accidents. Accident Analysis and Prevention, 11, 125-127.

Matthews, G. (1987a, June). Extraversion, self-report arousal and performance: The role of task factors. In D. Schalling (Chair), Extraversion/impulsivity. Symposium conducted at the meeting of the International Society for the Study of Individual Differences, Toronto, Canada.

Matthews, G. (1987b). Personality and multidimensional arousal: A study of two dimensions of extraversion. Personality and Individual Differences, 8, 9-16.

Neubauer, A. C. (1992). Psychometric comparison of two circadian rhythm questionnaires and their relationship with personality. Personality and Individual Differences, 13, 125-131.

O'Gorman, J. G. (1983). Habituation and personality. In A. Gale \& J. A. Edwards (Eds.), Physiological correlates of human behaviour: Vol. 3. Individual differences and psychopathology (pp. 45-61). San Diego, CA: Academic Press.

O'Gorman, J. G., \& Lloyd, J. E. M. (1987). Extraversion, impulsiveness, and EEG alpha activity. Personality and Individual Differences, 8. $169-174$.

Rall, T. W. (1980). Central nervous system stimulants: The xanthines. In L. S. Goodman \& A. Gilman (Eds.), Pharmacological basis of therapeutics (6th ed., pp. 592-607). New York: Macmillan.
Revelle, W. (1983). Factors are fictions, and other comments on individuality theory. Journal of Personality, 51, 707-714.

Revelle, W., \& Anderson, K. J. (1992). Models for the testing of theory. In A. Gale \& M. W. Eysenck (Eds.), Handbook of individual differences: Biological perspectives (pp. 81-113). Chichester, England: Wiley.

Revelle, W., Anderson, K. J., \& Humphreys, M. S. (1987). Empirical tests and theoretical extensions of arousal based theories of personality. In J. Strelau \& H. J. Eysenck (Eds.), Personality dimensions and arousal (pp. 17-36). London: Plenum.

Revelle, W., Humphreys, M. S., Simon, L., \& Gilliland, K. (1980). The interactive effect of personality, time of day, and caffeine: A test of the arousal model. Journal of Experimental Psychology: General, 109, 1 $3 !$.

Rocklin, T., \& Revelle, W. (1981). The measurement of extraversion: A comparison of the Eysenck Personality Inventory and the Eysenck Personality Questionnaire. British Journal of Social Psychology, 20. 279-284.

Schalling, D., Edman, G., \& Åsberg, M. (1983). Impulsive cognitive style and inability to tolerate boredom: Psychobiological studies of temperamental vulnerability. In M. Zuckerman (Ed.), Biological bases of sensation seeking, impulsivity, and anxiety (pp. 123-145). Hillsdale, NJ: Erlbaum.

Schulman, A. I. (1974). The declining course of recognition memory. Memory and Cognition, 2, 14-18.

Smith, B. D. (1983). Extraversion and electrodermal activity: Arousability and the inverted-u. Personality and Individual Differences, 4, $411-419$

Stelmack, R. M. (1981). The psychophysiology of extraversion and neuroticism. In H. J. Eysenck (Ed.), A model for personality (pp. 38-64). Berlin: Springer.

Stelmack, R. M., \& Geen, R. G. (1992). The psychophysiology of extraversion. In A. Gale \& M. W. Eysenck (Eds.), Handbook of individual differences (pp. 227-254). New York: Wiley.

Stenberg, G. (1992). Personality and the EEG: Arousal and emotional arousability. Personality and Individual Differences, 13, 1097-1113.

Strelau, J. (1987). Personality dimensions based on arousal theories: Search for integration. In J. Strelau \& H. J. Eysenck (Eds.), Personality dimensions and arousal (pp. 269-286). New York: Plenum.

Thayer, R. E. (1986). Activation-Deactivation Adjective Check List: Current overview and structural analysis. Psychological Reports, 58 , 607-614.

Underwood, B. J. (1978). Recognition memory as a function of length of study list. Bulletin of the Psychonomic Society, 12, 89-91.

Wilson, G. D. (1990). Personality, time of day and arousal. Personality and Individual Differences, 11, 153-168.

Zuber, I., \& Ekehammar, B. (1988). Personality, time of day and visual perception: Preferences and selective attention. Personality and Individual Differences, 9, 345-352.

Zuckerman, M. (Ed.). (1983). Biological bases of sensation seeking, impulsivity, and anxiety. Hillsdale, NJ: Erlbaum.

Received September 7, 1993

Revision received March 16, 1994

Accepted March 20, 1994 